\title{
12. COMMISSION DE LA RADIATION SOLAIRE ET DE LA SPECTROSCOPIE SOLAIRE
}

PrÉSIDENT: G. Righini.

Membres: MM. Abbot, H. D. Babcock, H. W. Babcock, Barabashev, Mlle Barbara Bell, MM. Brück, P. Carrasco, Chalonge, Das, d'Azambuja, Edlén, J. W. Evans, Evershed†, Gnevyshev, Goldberg, Houtgast, Labs, Locke, Melnikov, Menzel, Migeotte, Minnaert, Mlle E. A. Müller, MM. Mustel, Nicholson, Nicolet, A. K. Pierce, Plaskett, Rutllant, Schlüter, Schwarzschild, Severny, Sitnik, Smyth, Suemoto, ten Bruggencate, Thiessen, Tousey, Treanor, von Klüber, Waldmeier, Warwick.

\section{GENERAL}

This Report summarizes the research which has been made on solar radiation and solar spectroscopy from the end of I954 to the end of I957, as derived from publications and from personal letters by the members of the commission. Owing to the similarity between the subjects treated by this commission and those treated by Commissions Io, II and 13 , some duplication is unavoidable.

The published papers have been quoted here with their reference numbers given by the Extraits d'A stronomie du Bulletin Signalétique du C.N.R.S.; unpublished works have no reference numbers. The bibliography at the end of the Report has been suppressed; full information on the published papers can be obtained through their identification numbers.

Attention is drawn to the following books which should be of particular interest to research workers in the field of solar radiation and solar spectroscopy:

A. Unsöld, Physikder Sternatmosphären (Springer Verlag, Berlin, X955), second edition.

Les Molecules dans les Astres, edited by the Institut d'Astrophysique de l'Université de Liège-Cointe Sclessin.

\section{ENERGY WAVE-LENGTH CURVE}

A great effort has been made in the last three years to improve our knowledge of the far ultra-violet spectrum of the Sun, by employing several types of instruments fired to high altitudes by rockets. Tousey gave at the I955 Meeting of the I.A.U. a wide survey of the work performed in this field; after that date several successful flights supplied more material for study. Details of the flights and characteristics of the instruments are given by Tousey (16-52607). A revised analysis of the ultra-violet solar spectrum has been published by Wilson (I6-I6506); another paper by Tousey is to be published shortly. Other results have been published by Johnson and others (I7-I2432) (I7-25025), and by Jursa and others ( $17-37852)$. There is a general agreement on the small width of $L \alpha$ in emission. Some of the papers already quoted, and Byram and others (I8-9186) $(18-2576 \mathrm{I})$ found that the line is no wider than $0.3 \AA$, the limit imposed by the resolving power of the instruments. The opinion of Byram is that the intensity of the line is not associated with solar flares. The limb brightening of $L \alpha$ predicted by de Jager has not been found by Tousey and collaborators; on the other hand, Miller and others (I7-44II $)$ ( $18-43767$ ) have reached the conclusion that a slight limb brightening is present in $L \alpha$, possibly because of an appreciable increase of radiation in solar latitudes where the sunspot activity is higher.

Other emission lines have a distribution of intensity on the disk which are not exactly like that found by Tousey ( $16-52607)$; this may point to a dependency upon height in the solar atmosphere.

The distribution of the intensity of $L \alpha$ on the solar disk as obtained with a double monochromator carried by a rocket, is roughly similar to the distribution of the $\mathrm{Ca}$ II plages. 


\section{COMMISSION 12}

Photo-electric measurements of solar X-rays and ultra-violet radiation have been performed by Friedman ( $16-52618$ ) with photon counters and an ionization chamber covering selected regions between I and $I_{500} \AA$. The absorption of solar radiation in our atmosphere has been studied from results obtained in a dozen flights; besides, measurements taken during a small flare showed that the intensity of X-rays is high and persistent even after the decay of $L \alpha$. Measurements of the electron density of the D-layer from the absorption of cosmic noise, performed by J. Warwick and $\mathrm{H}$. Zirin, give only a $20 \%$ increase; an explanation of the contradiction between the two independent sets of observations might be found in a local production of X-rays in our atmosphere.

The intensities in the X-rays and ultra-violet spectrum of the Sun have been computed theoretically by Elwert (16-8782) and by de Jager (I7-12423) taking into account the coronal emission. Following Elwert (I8-I7630) (I8-35093), the X-radiation is due chiefly to the coronal condensations. The computations performed on the model of Waldmeier and Müller give results which are in agreement with the observations of Friedman.

From photo-electric observations of the comet Arend-Roland, Thiessen has found a strong correlation between the brightness fluctuations and the sunspot numbers formed by taking only the large groups into account. This indicates that an ultra-violet radiation is emitted only by large spot groups.

\section{CORPUSCULAR RADIATION}

The problem of the corpuscular radiation from the Sun has been treated anew by Vsekhsvyatsky $(16-63620)$, who has found that the yearly loss of the Sun amounts to ${ }^{10^{20}} \mathrm{~g}$ and that the active corpuscular stream near the Earth has a density of $10^{3}$ particles per cubic cm. Mustel (16-63620) answering to the criticisms of Vsekhsvyatsky confirms his earlier result that the mass leaving the Sun cannot exceed $6 \cdot 10^{16} \mathrm{~g}$ per year. An attempt to localize the sources of corpuscles on the Sun has been made by Sekido (r7-6r845); an analysis of the ionospheric effect and of the optical observations of solar phenomena has been performed by Ellison (I7-6r840). The pure observational aspect of corpuscular radiation has been studied by Newton (I6-6252I). Photo-electric investigations of the profile of the CaII K line have been performed by Smyth (16-63638) in order to detect some additional absorptions due to the clouds of particles; but no reliable result has been obtained.

According to Pecker, the emitting centres are located at a certain depth below the solar photosphere, for on the average the long-living $M$ regions rotate with the Carrington period. This result has been obtained by means of a correlation between the magnetic index $K_{p}$ and certain coronal features.

Theoretical researches on the problem after $195^{\circ}$ have been reviewed and criticized by Ferraro (16-526I7); Petukhov (I8-I7623) tried to explain the origin of solar corpuscular radiation by postulating the existence of powerful neutron sources on the Sun.

High-energy particles emitted from solar flares possibly originate in discharges with high current density; this is the opinion of Gold (18-889) who discusses the application of results obtained in laboratory experiments by Kurchatov.

Kawabata (17-6369), from an investigation of the motion of ionized clouds in the general magnetic field of the Sun, has deduced a simple model for the emission of lowenergy corpuscles.

In Kraushaar's opinion ( $17-1865 \mathrm{I}$ ) the presence of a galactic magnetic field can explain, under certain conditions, the emission of high-energy particles from a flare. The criticism of current theories concerning the origin of cosmic rays, made from an astrophysical point of view, leads Unsöld ( $16-58766)$ to the conclusion that cosmic rays possibly originate in a highly turbulent magnetic plasma. 


\section{RADIATION ET SPECTROSCOPIE SOLAIRE}

\section{VIOLET, VISIBLE AND INFRA-RED RADIATIONS}

The intensity distribution in the solar continuum between 3100 and $3800 \AA$ has been investigated by G. I. Thompson at Dunsink using a high dispersion concave grating spectrograph. A new determination of the intensity-wave-length curve of the Sun has been performed by Labs at the Pic-du-Midi Observatory. The photo-electric measurements of radiation from its centre cover the range $3300-7000 \AA$; the results, corrected for atmospheric extinction, have been compared with a standard carbon arc. The colour temperatures come out to be $6225^{\circ}$ on the violet side of the Balmer discontinuity and $7540^{\circ}$ on the red side; the Balmer discontinuity is $0.13 \AA$. A direct comparison of Sun and arc gave a brightness temperature of $6470^{\circ} \mathrm{K}$. Absolute measurements on the solar continuum have also been performed by Sitnik (I $8-35$ r20).

A report about the method of investigation and the results obtained for the infra-red solar spectrum have been published by Goldberg ( $16-58780$ ); wave-lengths of solar lines in the infra-red between II 984 and $25578 \AA$ are given by Mohler (I7-5IIII). A new photometric atlas of the solar spectrum between 2.8 and 23.7 microns has been published by Migeotte and others (I7-5IIOg). Measurements of solar radiation betwec 0.8 and 3.5 microns have been performed by Jaroslavcev (I8-52068). Significant progress has been made by extending the infra-red solar spectrum towards longer wave-lengths by optical methods. Sinton (I7-25026) has measured solar radiation at $\mathrm{I} .5 \mathrm{~mm}$ wave-length using a Golay detector in the focus of a large mirror. Theissing (I8-I7628) (I8-35104), employing a wire grating in front of a mirror and the Golay detector, has measured the spectral curve of the Sun in the millimetre wave-length range beginning at $0.9 \mathrm{~mm}$. Gebbie, using the interferometer technique and a Golay detector, has obtained very good tracings of the solar spectrum in the wave-length range 0.277 to $\mathrm{I} \mathrm{mm}$ with a resolving power of $0.2 \mathrm{~cm}^{-1}$.

\section{SOLAR CONSTANT}

A review of the corrections to be introduced in the measurements has been published by Georgi ( $16-16501$ ), Johnson (I6-526I9) and Tousey (I8-61274), taking into account the results of rocket observations, obtains a value of $2 \cdot 00 \mathrm{cal} / \mathrm{cm}^{2} \mathrm{~min}$ with a probable error of $2 \%$.

A search for variations of solar radiation in the visible range has been made by Hardie (17-37865), observing the magnitude of the planets Neptune and Uranus between I949-54: no significant variation has been found.

Studies of solar constant variations and weather forecasting have been performed by Abbot on the basis of the pyroheliometric measurements of the solar radiation.

The colour of the Sun has been measured by Nikonova ( $17-37866)$, taking as comparison the brightest stars. Karandikar $(16-63656)$ has found that the 'luminance' of the centre of the disk is 243000 candles $/ \mathrm{cm}^{2}$.

\section{LIMB DARKENING}

The measurements of Peytureaux quoted in the preceding report have now been published (I6-52605). The same author has measured the limb darkening of the Balmer discontinuity (16-22783). The darkening at the extreme limb has been measured by Scheffler (16-63657) during a partial eclipse. By correcting for the influence of the absorption lines he has found the true intensity profile of the solar limb at $3760 \AA$. A similar research has been performed by Lamla (r8-9rI) at the I954 partial eclipse. Warzée (16-52604) has solved the integral equation of the limb darkening in a new way after a critical study of the current methods. A model of the upper photosphere has been established by Hubenet and de Jager (18-52060) on the basis of results obtained from eclipse photometry of the solar limb. A comparison between observations and theoretical results obtained from different models has been performed by Swihart (I7-44IO8). 


\section{COMMISSION 12}

\section{MEASUREMENTS OF WAVE-LENGTHS. SOLAR ROTATION}

Interferometric measurements of selected solar lines at $6500 \AA$. has been performed by Adam (I8-900), and the red shift of all lines has been deduced. The results show that there is a linear relation between the red shift divided by the wave-length and the logarithm of the equivalent width. These results agree with those obtained at the Allegheny Observatory in I925-27.

The whole observational material has been recently discussed by Schröter (I7-250I4) (18-6I265) who has explained the differences between the red shift observed and the relativistic one by supposing that in the solar atmosphere strong local temperature fluctuations are present.

A new empirical approach to the problem of the solar red shift based on re-analysis of all existing observational material has been presented by Freundlich and Forbes (18-68033) (r8-68034), who have found that the red shift is well represented with a twoterm formula: one term is dependent on all causes which may be influencing the Sun-arc wave-length differences, while the other is independent of the terrestrial standards, and is well represented by a geometrical function which is closely related to a secant law but which takes into account the curvature of the solar layers. The constant of proportionality has been shown to be temperature-dependent and it has the significance of an 'interaction-term'.

The red shift of six infra-red lines belonging to two multiplets measured by L. Herzberg (I8-9I88) seems to indicate the existence of a term dependency in the displacements.

New measurements of the solar rotation made by Milosevich ( $16-36144)$ (16-63655) observing the positions of small chromospheric flocculi indicate that the velocity is constant during a solar cycle.

Safronov (I7-6I827), discussing the emission of matter from the Sun, found that the Sun could acquire its present rotation as a result of infall of the matter from protoplanetary clouds, only if its loss of mass by radiation (photon or particles) in the past would have been $10^{3}$ times the present photons radiation.

\section{IDENTIFICATIONS AND ABUNDANCES OF ELEMENTS AND COMPOUNDS}

A redetermination of the abundance of forty elements in the solar atmosphere has been performed by Goldberg, Aller and Müller (16-63613) on published data supplemented by infra-red data and by measurements made with the vacuum spectrograph of the MacMathHulbert Observatory. Greenstein and de Jager (18-52067) have investigated the possible occurrence of Technetium in the Sun, finding that there is at present no reliable evidence for its presence.

Severny, studying the profile of $40 I_{9} \cdot \mathrm{r}_{4} \AA$, deduced that the abundance of $T h$ is of the order of $10^{10}$ atoms per gram of solar material: thus the solar ratio $\mathrm{Th} / \mathrm{Pb}$ is approximately a hundred times smaller than that of the Earth.

The long-debated question of the existence of deuterium in the solar atmosphere is still not completely solved. Severny, studying the profiles of some lines near $6560 \AA$. concluded that the abundance of $\mathrm{D}$ must be of the order of $10^{-5}$ times that of hydrogen; furthermore he found that the features which indicate the presence of $D$ are strengthened in 28 grains showing 'moustaches'. His opinion is that $\mathrm{D}$ and $\mathrm{Li}$ abundances cannot be brought into agreement with the hypothesis of the incomplete mixing unless peculiar processes occur in the outermost layers of the Sun.

A search for D performed by Kinman ( $18-35$ I02) at Oxford has given a negative result. Severny, criticizing this work, remarks that the resolving power of the instrument was not sufficient, and that the content of water vapour at the time of observation was too high.

Following a suggestion by Fowler that $\mathrm{D}$ may be produced in flares by low energy reactions, Goldberg, Mohler and Müller measured some $\mathrm{H} \alpha$ profiles in a limb flare of I956 February 10. All profiles showed a marked asymmetry between $I \cdot 5$ to $2 \cdot 0 \AA$. to the 


\section{RADIATION ET SPECTROSCOPIE SOLAIRE}

violet side, which can be produced by $\mathrm{D}$ as well as by a peculiar distribution of velocity in the emitting gases.

The abundance of the isotope ${ }^{13} \mathrm{C}$ has been measured by Righini (18-68036) on photoelectric tracings of the solar spectrum taken at the McMath-Hulbert Observatory. The ratio ${ }^{12} \mathrm{C} /{ }^{13} \mathrm{C}$ turns out to be around 10,000 .

A general discussion of the abundances of $\mathrm{Li}, \mathrm{Be}$ and $\mathrm{D}$ on the solar atmosphere has been published by Shklovsky (I7-37846).

A review of the problem of molecules in the solar spectrum has been given by Broida (I8-68032) in his introductory report at the Liège meeting of I956. A revision of the identifications of rotational lines belonging to $\mathrm{CH}, \mathrm{OH}$, and $\mathrm{CN}$ has been made by Moore (I8-68035). Identifications of solar CN lines based on new laboratory measurements have been made by Genard (I7-44II6). A very important contribution to a better knowledge of the infra-red solar spectrum has been given by Migeotte and others (I7-44II8); more extensive identifications are contained in the infra-red atlas of the solar spectrum ( $17-5 \mathrm{IIO0})$.

Telluric lines of $\mathrm{H}_{2}{ }^{18} \mathrm{O}$ and $\mathrm{H}_{2}{ }^{17} \mathrm{O}$ have been identified by Benedict(I8-6803 $\mathrm{I}$ ) in the 6-micron band of the solar spectrum.

\section{INTENSITY AND PROFILES OF FRAUNHOFER LINES}

Preliminary results on the profiles and detailed structure of some lines are presented by McMath and collaborators (I8-9I9I). Local Doppler displacements have been measured on spectra taken with the new vacuum spectrograph. Profiles of seventy lines obtained photo-electrically by Rogerson (18-61264) suggest the existence of a constant turbulence in all layers of the solar atmosphere. An investigation on faint lines has been performed by Zalubas (18-904). Mohler and Goldberg (I8-9I92) investigated the origin of the widening of infra-red lines, trying to separate the turbulence from the pure thermal broadening.

Following De Jager (I8-9I89), it is necessary to take into account the detailed structure due to the Stark effect, if satisfactory agreement with the observed profiles of the Brackett alpha line is to be obtained.

Calculations performed by Severny on a de Jager model show that the absorption coefficient due to neutral $\mathrm{H}$ is larger than that due to Stark effect. This is valid for $\mathrm{H} \alpha$ since for $\mathrm{H} \beta$ the Stark effect is the more important; in addition, he has found good agreement between the calculated and observed profiles of $\mathrm{H} \alpha$. A comment on the Stark broadening of $\mathrm{H} \alpha$ in the Sun is given by Doherty $(\mathrm{I} 6-636 \mathrm{II})$, who discusses the theories of Holtsmark and Kolb.

The problem of the influence of temperature inhomogeneities in the profiles of the hydrogen lines is dealt with in detail by de Jager (I6-636r4); the central intensity and the Doppler core of infra-red Ca II lines are discussed by Miyamoto who explains the high residual intensity in terms of metastability in the lower level and the strong interlocking with the $\mathrm{H}$ and $\mathrm{K}$ lines through the upper level.

Very accurate measurements of the central intensity of the $\mathrm{D}$ lines have been made by Scrimger and Hunten (1 $7-37854$ ) using a Sodium absorbing tube in order to correct for the influence of scattered light.

The blending effect on the intensity of a weak line due to the wing of a strong line has been investigated by Rauer (16-63592); observations of weak lines perturbed by the CaII $\mathrm{K}$ line are in agreement with theory.

Interferometric investigations performed by Hindmarsh (I7-5II07) on the structure of solar lines show that some profiles are affected by hyperfine structure and this has to be taken into account in order to obtain a correct interpretation of the observations.

An attempt to estimate the intensities of an infra-red forbidden doublet of $\mathrm{Ca}$ II in absorption and in emission has been made by Nikitin (16-2278I).

Researches on a latitude effect in Fraunhofer lines have been performed by Das, measuring the equivalent widths of $g$ and $K$ lines at the poles and at the equator of the 


\section{COMMISSION 12}

Sun: the result is that the temperature at the pole is about $90^{\circ}$ higher than that at the equator. A similar result has been obtained by Beckers, measuring the equivalent width of selected lines at different latitudes.

\section{Centre-limb variation of Fraunhofer lines. Curves of growth}

The behaviour of absorption lines at different positions on the solar disk has been investigated by the following authors.

Bray (18-61263) investigated the D lines photographically.

Elste (I7-I243I) by employing a Lummer plate, measured the profiles of some average intensity lines: the observed profiles have been compared with the theory.

Godoli observed four $\mathrm{Fe} I$ lines in the $\mathrm{I} \cdot 6 \mu$ region, and found that the damping constant is about 2000 times the theoretical one: he also found evidence for an increase of turbulence with height.

Pagel (I8-9I93) observed the equivalent width of some infra-red lines.

Sankaranarayanan measured the centre to limb variation of fifty-five lines of $\mathrm{Ti}$, and found no evidence of a maximum at $40 \%$ of the solar radius.

Stefanovich investigated the run of equivalent width of rare earth lines, finding pronounced deviations from the results for other lines.

Suemoto performed interferometric measurements of line width with a resolving power of more than a half million, finding, after correction for the saturation effect, that faint lines are wider than the stronger ones: the widths increase slightly toward the limb, suggesting a circulation which is presumably associated with the granulation.

Waddell observed photo-electric profiles of many lines belonging to Fe I, Ti r, Va I. From the run of the centre to limb variation he deduced the existence of a non-isotropic turbulence having a radial velocity of $1.8 \mathrm{~km} / \mathrm{sec}$ and a tangential component of $3.0 \mathrm{~km} / \mathrm{sec}$.

Pecker (18-68023) investigated the centre to limb variation of some molecular bands, and has concluded that rotational lines give some indication of the physical state of the low chromosphere.

Voigt ( $16-22773$ ), after studying the central intensity of two $\mathrm{Fe}$ I multiplets, reached the conclusion that it is necessary to take into account the inhomogeneities of the temperature in order to have a better agreement with the solar models.

Hitotuyanagi (I7-250I2) gave a modified model of the Aller and Pierce solar atmosphere in order to explain the centre to limb variation of the $\mathrm{D}$ lines.

Wehlau (18-25762) proposed a new explanation of the behaviour of the centre to limb variation of solar line widths in terms of a variation with depth in the velocity of turbulence.

The centre to limb variation of the curve of growth for 150 weak and medium intensity lines of $\mathrm{Ti}, \mathrm{Va}$ and $\mathrm{Cr}$ has been studied by Bretz ( $\mathrm{I}_{7}-3 \mathrm{I} 327$ ), who found that the observations can be explained only if the ionization in the upper photospheric layers is higher than that required by equilibrium conditions.

Detailed computations on the curve of growth of many lines have been performed by Inaba (I $7-6378)$ who takes into account the depth of formation of lines and the gradient of temperature in the atmosphere.

The curve of growth of Balmer lines has been computed by C. Pecker (r6-52579); and the classical theory has been improved by J. C. Pecker, taking into account the deviations from equilibrium. New formulae are given for the weight-functions in conditions deviating from local thermodynamical equilibrium; it follows that the curves of growth corresponding to different multiplets differ in the height of the flat portion. This fact may require a revision of the abundances, and of the excitation temperatures; the abundance of $\mathrm{Ti}$, for instance, has to be multiplied by a factor of Io. The ionization equilibrium is also affected. 
THEORY OF LINE FORMATION. MODELS OF THE SOLAR ATMOSPHERE

Formulae for the computation of the intensity of weak spectral lines have been established by Barbier (I8-680rI), following the methods of weight-functions. Corrections to the pressures in solar models have been introduced by Minnaert in order to account for the helium and metal content of the atmosphere; the precision of this method has been investigated by Hubenet (I8-890).

The formation of absorption lines by coherent and non-coherent scattering has been investigated by Inaba, introducing the line absorption to continuous absorption coefficient and the temperature distribution of the model.

J.C. Pecker has found that the titanium lines in the solar photosphere are not formed in conditions of thermodynamical equilibrium. The deviations from equilibrium conditions are smaller for the levels having high excitation potentials; the temperature inhomogeneities of the atmosphere have no influence on this phenomenon.

A new theory on the formation of absorption lines has been advanced by Plaskett (I7-5IO82); the source function and the absorption coefficients are calculated starting from the transition probabilities and taking into account the values of kinetic excitation and ionization temperatures.

The theory of the polarization of Fraunhofer lines has been investigated by Jäger for the case of line cores formed mostly by coherent scattering; taking into account the influence of magnetic field on the incident light which is partially polarized, Jäger finds full agreement with his new polarization measurements on $\mathrm{Ca} 4277$ at centre and at the limb.

The model of the solar atmosphere proposed by Böhm (I6-58774) has been further discussed by Weidemann ( $16-22774$ ), who deduces the abundances of some elements and the distribution of temperature. The resulting metal abundances are smaller than those determined by Claas. Following Weidemann ( $16-58778$ ), the damping given by this model is not always in agreement with observations.

Voigt (I8-903) found that the asymmetry of the oxygen infra-red triplet may be well explained by Böhm's model if the temperature inhomogeneities decrease in the upper part of the photosphere.

Methods for computations of the central intensities and of the profiles of absorption lines have been developed by ten Bruggencate (I7-6I828), taking as his integration variable the logarithm of the optical depth.

A procedure for the empirical determination of the absorption coefficient, which has the great advantage of being independent of any model, has been proposed by Goldberg. The method requires the accurate observation of the profile of pairs of lines whose relative multiplet strengths are known; its application to a pair of lines observed by Suemoto has immediately given a Doppler width which is slightly smaller than that obtained by Suemoto.

A model of the upper photosphere has been obtained by Hubenet and de Jager rediscussing the eclipse observations of Heyden (I952); a model which corresponds closely to the one deduced from the continuous spectrum has been obtained by Pecker studying the spectrum of the element $V_{I}$; however, the centre to limb variation of the molecular bands gives a suitable model for the lower chromosphere.

Schwarzschild (I8-6I258) proposed as the most suitable for explaining the observations of the photosphere, the composition of the Sun, and depth of convective zone, a solar model with convective envelope and inhomogeneous interior.

\section{GRAN ULATION}

Our knowledge in this field has made notable progress, thanks to the pictures of the Sun taken from balloons. Dollfus and Blackwell, carrying a telescope in an open balloon to the height of $20000 \mathrm{ft}$, succeeded in obtaining very good pictures of a small part of the solar disk. The granulation shows some fine structure inside the granuli but the overall dimension is of the order of $I^{\prime \prime}$. 


\section{COMMISSION 12}

Schwarzschild has organized a unmanned balloon carrying a I2-inch solar telescope which was photo-electrically guided on the Sun. Two flights have been performed at a height of $25000 \mathrm{~m}$ and 8000 exposures have been taken for each flight with an exposure time of o:oor.

The granulation shows a very fine structure - up to recently unresolved-and a cellular character which appear to be irregular; the dimensions of the granulation elements are scattered over a wide range.

Investigations of the turbulence in the solar atmosphere have been performed by Uberoi ( $17-37850)$ ( $16-63619)$ who has succeeded in finding that the characteristics observed in the solar granuli are almost entirely determined by the conditions of observation; by Frenkiel ( $16-52606$ ) (16-63612), who defined a turbulence spectrum in order to describe the characteristics of the granulation. Besides, he performed some observations on one plate showing exceptionally good definition in the far red.

The influence of the solar cycle on the number of granules has been investigated by Macris (I7-639I), who found a positive result.

The discovery of the fluctuations of intensity in the Fraunhofer lines (wriggled structure), made through the excellent performance of the McMath vacuum spectrograph, has thrown new light on the relationships between the structure of the low chromosphere and the granulation. The observations made by McMath and collaborators (I7-37853) of Doppler shifts in the solar granules has led to the discovery of connexion between the granulation and the structure of the low chromosphere [Goldberg and others (I 8-52066)]. In fact, the fluctuations observed in $\mathrm{H} \beta$ are associated with displacements towards the violet in the metallic lines, and this correlation has been recently confirmed from a study of more material taken with the vacuum spectrograph. Similar researches on the granulation have been performed by von Klüber (18-9190), who has also found a connexion between the fluctuation in the intensity of Fraunhofer lines and the granulation itself.

It seems that the r.m.s. velocity deduced, from iso-photometric tracings of wriggled lines taken at several points on the disk, is of the order of $0.4 \mathrm{~km} / \mathrm{sec}$ and increases toward the limb. Local fluctuations from point to point on the disk have been observed and significant differences from one line to the other are evidenced (Goldberg). Besides, stronger lines show a progressive increase in the r.m.s. velocity from 0.6 to $I .5 \mathrm{~km} / \mathrm{sec}$

A theoretical investigation of the ratio between the horizontal and the vertical average velocity in the photospheric convection, performed by Unno at Princeton, leads to the conclusion that the observed excess of the horizontal over the vertical velocity is a simple consequence of the photospheric density gradient.

\section{SUNSPOTS}

Equivalent widths of $\mathrm{Cr}$ I, Fe I and $\mathrm{Ti}$ I lines in sunspot spectra have been measured by Abhyankar (I7-I2430) and others; from the curve of growth excitation temperatures of $4030^{\circ}, 4200^{\circ}$ and $3800^{\circ}$ have been obtained. Laborde (I 8-43766), investigating the molecular bands in spots and photosphere has confirmed the identification of $\mathrm{MgH}$ in the former. Sunspot spectra have been taken at Göttingen by ten Bruggencate and collaborators with a concave grating spectrograph. The spectra, which cover the range between 3900 and $7900 \AA$, will be used for centre to limb variation of spot spectra. Photo-electric measurements of the monochromatic brightness of the penumbra by Makita and others at the Tokio Solar Tower are presently in progress. The scanning of the solar image is performed with a speed which is much higher than the average speed of the scintillation.

The granulation observed by Thiessen in the umbral region of the sunspots has been confirmed photographically by Rösch. In Thiessen's opinion the presence of the granulation in sunspots, the Evershed effect, the magnetic field and electric conductivity in sunspot regions, all need to be re-discussed, since too many anomalies exist. 


\section{RADIATION ET SPECTROSCOPIE SOLAIRE}

Atomic absorption coefficients for Zeeman split components have been studied by Stephanov in the case of an arbitrary angle between the direction of the magnetic field and the line of sight. Application of the theory to Fe 6I73 in sunspots shows that the equivalent width increases by $25 \%$ in a field increasing from 0 to 2500 gauss and for an angle of $75^{\circ}$. Besides, the mean turbulent velocity in sunspots obtained from sigma components results as $I .8 \mathrm{~km} / \mathrm{sec}$.

The same problem has been investigated by Unno making use of the method of Stokes' parameters. He also suggests a method of determining the orientation of the magnetic lines of force inside a sunspot.

The magnetic intensification of the lines and the effect on the curve of growth have been studied by J. Warwick (16-52608); the basic matrices for the detailed transfer theory in sunspots and the solution for a source function of practical importance are given by the same author in another paper.

\section{MAGNETIC FIELD}

The investigation of the general magnetic field has been continued with success by Babcock (16-63637) with the magnetograph. More than 800 tracings have been obtained up to now, from which fine detail in intensities higher than 0.3 gauss can be observed. From these observations it appears that the general magnetic field is a dipole field whose mean intensity is I gauss. The polarity is opposite to that of the Earth and it is limited to latitudes higher than $\pm 55^{\circ}$. The main features observed in the lower latitudes are the $\mathrm{BM}$ regions, which correspond generally to CaII plages; and UM regions, which Babcock suggests may be identified with the hypothetical M region of Bartels. There is some evidence that the UM regions may be correlated with the 27-day sequential geomagnetic disturbances, and following Simpson (I7-I8652), with the increase of primary cosmic radiation intensity.

A second magnetograph is now in operation at the Mt Wilson Observatory. The installation of a new magnetograph at the Crimean Observatory has been reported by Severny, Nikulin and Stepanov; with this instrument fine structures of the weak magnetic fields have been observed even in regions of $5 \times 5$ seconds of arc.

A general report of all observational methods used for the purpose of observing the Sun's magnetic field has been written by von Klüber for Vistas in Astronomy.

Regular observations of the magnetic field in spots are performed at Mt Wilson, the Crimean and the Potsdam Observatories.

The Mount Wilson magnetic observations of sunspots, giving spot position, as well as magnetic field strength and polarities, are being reproduced on microfilm. These daily records cover an interval of more than 35 years.

A model of the general magnetic field of the Sun has been presented by Alfvèn (I7-5IOgI) from information obtained through the observations of cosmic rays and geomagnetic disturbances. Such a model is in agreement with the structure of the corona. The opinion of Mogilevkij (18-17622) is that the general magnetic field of the Sun has to be determined with direct astrophysical methods only, since indirect methods are too uncertain.

Theoretical work on the general magnetic field has been published by Conway (I8-9I77). Following Alfvén (I8-3509I), the magneto-turbulence of the photosphere can sometimes strongly influence optical measurements of the magnetic field: one such case is discussed in the paper quoted above.

\section{CHROMOSPHERE}

The structure of the low chromosphere has been investigated by Müller, Mohler and Goldberg from the photo-electric profiles of $\mathrm{H}$ and $\mathrm{K}$. It has been found that the central intensities decrease slightly toward the limb because they are formed in a region of the 


\section{COMMISSION 12}

chromosphere where the excitation temperature is nearly independent of the height. The Doppler widths also increase towards the limb.

The behaviour of $\mathrm{H}$ and $\mathrm{K}$ over the spots has been studied by Mustel (I7-44I07), who observed that in such cases the emission components of both lines are narrower and the central absorption component disappears completely.

Mitropolskaja (I7-5IIIo) has published some measurements of the profiles of $\mathrm{H} \alpha$ and $\mathrm{H} \beta$ in the plages, and the curves of growth of some multiplets (17-31333). The central intensities of Balmer lines have been studied by Böhm-Vitense ( $16-58779$ ); some fine structures in solar spectra-notably on plages, eruptions, etc.-have been observed by Severny (18-68037) with a high resolving power and a dispersion of $\mathrm{ro} \mathrm{mm} / \AA$.

Uberoi's method of the correlation pattern has been applied by Jefferies (I7-3I332) to the granulation observed on $\mathrm{H} \alpha$ photographs taken with a Lyot filter. The spatial correlation becomes negligible for distances larger than $3 \times 10^{4} \mathrm{~km}$. Rogerson (16-526I3) has investigated the fluctuations of intensity on $\mathrm{H} \alpha$ and $\mathrm{Ca}$ II spectroheliograms. Following de Jager, the fine mottling observed in $\mathrm{H} \alpha 3$ spectroheliograms is identified with the spicules seen from above; whereas the coarse mottling, which is visible in the wings of $\mathrm{H} \alpha$, is produced by a combination of a shift of $\mathrm{H} \alpha$ and a widening of the line. It seems that the widening is correlated with a red shift.

The spectrum of the chromosphere at the limb without an eclipse has been observed by Roberts (I8-9I99) with the coronograph; also by Rauer who took $\mathrm{H} \alpha$ spectra in the third order of a concave grating spectrograph. The latter set of observations refer to heights up to $7000 \mathrm{~km}$; a self-absorption effect is visible up to $5000 \mathrm{~km}$. $\mathrm{H} \alpha$ profiles at several heights in the chromosphere up to $12500 \mathrm{~km}$ have been obtained by Elske Smith with the large spectrograph of the Sacramento Peak Observatory.

Spectrophotometric researches on the $\mathrm{H} \alpha$ profile in the spicules have been performed by Michard (I7-44I24) and (I8-9I98).

With a Fabry-Perot interferometer coupled with a low-dispersion spectrograph, Treanor (I7-25027) has obtained high resolution spectra of some chromospheric lines.

The temperature and the turbulence of the chromosphere have been obtained by Redman (I6-6367I) from the profiles of strong emission lines belonging to high-dispersion eclipse spectra. From the CN bands Thomas (I8-68025) and Pecker (I6-63670) have deduced the temperature of the low chromosphere, using spectra taken during the I952 eclipse. The behaviour of some flash lines and the photometric profile of the limb have been obtained by Kristenson ( $16-58787$ ) from a set of cinematographic exposures taken during the I945 eclipse.

A calibration of the empirical intensity scale of the flash lines given by Mitchell, has been made by Namba (I7-37858) taking advantage of the observations of PannekoekMinnaert and Cillié-Menzel.

Three new models of the solar chromosphere have been made by Athay and others (16-22770), F. Böhm-Vitense (16-28775) and Piddington (I6-I6500). From the analysis of the results of the 1952 eclipse, Athay has deduced that the chromosphere is composed of three regions whose temperature and ionization are different; whereas Böhm-Vitense gives a model with a continuous distribution of temperature and ionization deduced from the observations of Menzel and Cillié. Piddington, having criticized the presently accepted models, introduced a new model which satisfies both radio and optical observations. The requirements of a chromospheric model have been later discussed by Athay and others (I7-6I825), and in a further paper the temperature gradients in the chromosphere have been investigated (I7-6I824) (I7-I8649).

Deviations from thermodynamical equilibrium in the chromosphere, under various hypotheses of Stark and Doppler broadening, are investigated by C. Pecker and others (I7-37844) (r7-37845) from a study of the behaviour of Balmer lines; the populations of the levels $n=\mathrm{I}$ and $n=2$ were computed by taking into account the spicular structure of the chromosphere. The run of the electronic temperature and of the radial component of turbulence with height has been computed by de Jager. 


\section{RADIATION ET SPECTROSCOPIE SOLAIRE}

The intensities of the Lyman lines emitted by an isothermal atmosphere of pure hydrogen have been calculated by Jefferies (I6-29I78) for several models with temperatures ranging from $10^{4}$ to $2 \cdot 5 \cdot 10^{5}{ }^{\circ} \mathrm{K}$; and this has led to more satisfactory values than before. Athay and others ( $18-43765)$ have derived a new chromospheric model which is capable of reproducing the intensity of Lyman-alpha observed. Plaskett (I8-61257), from a study of the presence of Lyman-alpha in emission on the solar disk, has deduced that the photospheric radiation cannot be the sole cause responsible for the excitation of the chromospheric spectrum, but that other mechanisms have also to be present. Oster (I7-6I826) has postulated the existence of a transition layer with abnormal excitation conditions between the chromosphere and the corona.

The Helium problem has been investigated by Jefferies (I7-2500I), who has computed the populations of some He levels for temperatures ranging from $10^{4}$ to $10^{5}{ }^{\circ} \mathrm{K}$; by Zirin (I8-89I); by de Jager who, performing the computation of the He flash spectrum on the basis of the two-elements chromospheric model, and taking into account the deviations from equilibrium, has found that the $\mathrm{H}$ I and $\mathrm{H}$ in lines can be explained on the assumption of a temperature of $10000^{\circ} \mathrm{K}$ for the hot elements at a height of $2000 \mathrm{~km}$. The problem of the He excitation and the inhomogeneity of the chromosphere has also been studied by Krat (I7-5II08).

The calculation of the acoustic energy produced by the Sun's convective layer, and the influence of this energy on chromospheric turbulence, has been performed by Unno $\left(r_{7}-6383\right)$, who has also interpreted the spicules on this basis. The stability of the turbulence of a magnetic field has been investigated by Mitaka.

Spectra of the great flare of I949 August 5 have been studied by Mustel and Severny (I7-3I335), and various interpretations of the emission of the continuous spectra in solar flares have been given by Mustel (I7-5I093).

\section{PROMINENCES AND CORONA}

High dispersion spectra of prominences have been obtained by Zirin (I7-5III2); Haupt ( $17-5$ II06) has identified twenty-two emission lines on one plate taken with the coronagraph. Ivanov-Kholodnyj ( $17-5 I I I 6)$ has made a comparative study of the $\mathrm{H}$ and $\mathrm{He}$ lines in many quiescent prominences. The same author (17-31330) has photographed some prominences on the infra-red line $\mathrm{He}$ ro830 and compared the intensity of this line to that of $\mathrm{H} \alpha$ and $\mathrm{D}_{3}$.

Prominence spectra have been taken by ten Bruggencate with a concave grating spectrograph, with a view to studying the profiles of many lines; since these spectra show the $\mathrm{H}, \mathrm{He}$ and $\mathrm{Ca}$ II infra-red lines, it will be possible to separate the effect of turbulence from that of the temperature.

The possibility of deriving the physical conditions of a prominence from the emission lines after correction for the self-absorption has been discussed by Severny (I7-37860); the temperature and equilibrium of hydrogen in a prominence has been investigated by Zirin (r8-25772). From observations of $\mathrm{H} \alpha, \mathrm{H} \beta$ and $\mathrm{D}_{3}$ he has found that some prominences which are embedded in bright coronal regions may have temperatures ranging from 25000 to $200000^{\circ} \mathrm{K}$. The same problem has been discussed by Jefferies (I8-35094) from a comparison of the observed profiles of $\mathrm{H} \alpha$ and those calculated on the basis of a simple model which takes into account the emission of the prominence and the scattering of the photospheric radiation. He has deduced the temperatures and the electron pressures in the prominences observed.

The polarization of the prominence lines has been calculated by $\mathrm{J}$. Warwick, taking into account the multiplet scattering and the effect of the stimulated emission on the populations in the upper level of the line.

The brightness variation of the corona and its relationship with the solar cycle have been studied by Abbot ( $17-6396)$; a similar investigation on the monochromatic corona has been performed by Waldmeier ( $16-8802$ ), who found a double period of activity in a simple cycle. The electron temperature of the corona, as deduced from the ratio of 


\section{COMMISSION 12}

Fe $\mathrm{x}$ and $\mathrm{Fe} \mathrm{xIV}$ lines, shows a difference between the northern and southern hemispheres [Petri (16-4340I)]; furthermore, in the spot region, there is a maximum of the red coronal line.

A study of the widening of coronal emission lines in the active regions of the sun has been performed by Billings (16-22789); the temperatures obtained from the line widths have been compared with those deduced from the intensities (18-905). The exact width of the coronal lines has been determined interferometrically by von Klüber and Jarrett (I8-9I9) at the I954 eclipse; a local fluctuation of temperature seems to be present in some parts of the corona.

Applying an extrapolation method to the study of the coronal lines, Naqvi (I6-52578) gives for the line $5694 \AA$ the probable identification of A xv. The identification of the line $5446 \AA$ given by Edlcn seems to be confirmed by the spectrophotometric measurements of the profiles made by $C$. Pecker and others $(16-34400)$. The classification of both lines 5446 and $5694 \AA$ is again discussed by Rohrlich (I8-877) and Layzer (I8-43748).

The physical conditions inside a coronal condensation have been investigated through a study of intensities in the continuum and in a few lines by Waldmeier (I8-2577I) who has deduced the ionization temperature and the abundance of Fe.

Two papers by Roberts (I8-25774) and Notuki ( $17-25036)$ deal with the radiations emitted by the coronal plasma. Following Roberts the ultra-violet radiation and the $\mathrm{X}$-rays are produced in the coronal condensations where the temperature is very high. Notuki has discussed the geomagnetic perturbations and the corpuscular radiations emitted by the active centres of the corona.

\section{INSTRUMENTS}

The vacuum spectrograph of the McMath-Hulbert Observatory has been described by McMath (I7-44078), who has given some examples of the performance of this instrument. Severny (I7-5 I050) has described the new solar tower of the Crimean Observatory and has compared his results with others obtained with similar instruments. A new type of solar telescope has been erected in Oxford by Plaskett (I8-9116), which consists of a Cassegrain telescope equatorially mounted and having an equivalent focal length of $35 \mathrm{~m}$.

At the Göttingen Observatory the new spectrograph has been tested with a mercurykrypton lamp. The theoretical resolving power is almost obtained, thanks to the low level of scattered light. For photo-electric work on the solar spectrum a photo-electric photometer has been mounted, which can equally well give ratios or differences of intensity. In addition, a new concave grating spectrograph has been set up for the study of prominence and spot spectra. The range covered is between 3900 and $8600 \AA$ : the setting on the slit is made by means of a Lyot filter.

A solar spectrograph for the study of the active zones on the Sun, of prominences and eruptions, has been installed at Arosa by Waldmeier. It has a plane grating and a focal length of $12.5 \mathrm{~m}$. The image has a diameter of $29 \mathrm{~cm}$ and the telescope has an $f$-ratio of I/I IO.

Migeotte reports that a large prism-and grating-spectrograph is now under construction at Liège for the Jungfraujoch Observatory, where it will be installed probably at the end of 1957 . The spectrograph is a $7 \mathrm{~m}$ Ebert-type vacuum instrument which will be equipped with an original Babcock grating. This spectrograph is designed for work in the ultra-violet as well as in the infra-red, using either photographic or photo-electric detection. It will be used mainly for solar spectroscopy in conjunction with a solar image of $\mathrm{I} 2 \mathrm{~cm}$.

The 'heliographe monochromatique' of Lyot has been described by Grenat ( $16-43359)$; a new solar eye-piece using a pentagon prism has been constructed by Magun ( $17-24966$ ).

Two new magnetographs are now in operation: one in Cambridge (England)described by Beggs (18-43718)-can record field intensities down to I gauss; the other at the Crimean Observatory has been made by Nikulin, Severny and Stepanov. The 


\section{RADIATION ET SPECTROSCOPIE SOLAIRE}

mean noise level of the second is 0.35 gauss but during good atmospheric conditions it can record fields down to 0.1 gauss. An automatic device for the compensation of the line-of-sight velocity has been included in this instrument.

A balloon-borne system for tracking the Sun with a precision of $15^{\prime}$ is described by Edwards (18-25720). The performances obtained in two flights up to $30 \mathrm{~km}$ are given. Boyd (I8-90I) gives a complete description of many instruments utilized for rocket investigations of solar radiation at high altitudes.

The observation of the $\mathrm{K}$ corona is now possible, thanks to a new coronameter constructed by Wlérick and others (18-52033).

Due to the very low level of scattered light and of instrumental polarization, the polarization of the $\mathrm{K}$ corona can be measured photo-electrically.

A new interference filter has been constructed by Gil'varg (I7-24967).

A study of the instrumental conditions suitable for the reduction of temperature fluctuations inside a telescope has been performed by Rösch (I7-6335) at the Pic-du-Midi Observatory. Rösch suggests that by filling the tube with He the temperature fluctuations will be smoothed out.

A very rapid electronic instrument for the determination of isophotal contours has been described by Dennison (17-12396).

\section{SPECTROSCOPIC DATA OF ASTROPHYSICAL INTEREST}

The relation between the computed and observed values of oscillator strengths has been discussed by Allen and others (r8-9r59) for the elements $\mathrm{Ti}$ and $\mathrm{Ni}$. The same values have been computed by Nikitin $\left(\mathrm{I}_{7}-6365\right)$ for some transitions of $\mathrm{Ca}$ II which are of astrophysical importance. A set of physical parameters for iso-electronic sequences in the atoms involved in the emission of coronal lines have been computed by Zirin ( $16-63574)$ (I7-I24IO) and by Layzer (I7-I2408).

The molecular absorption coefficient of the $\mathrm{A}$ band of $\mathrm{O}_{2}$ has been measured by de Jager (18-52046), who has obtained a value of $I \cdot 7$ times that derived from an analysis of the solar spectrum.

Laboratory transition probabilities in the Swan band emitted by the $\mathrm{C}_{\mathbf{2}}$ molecule have been determined by Phillips (18-61264); also, vibrational transition probabilities for many molecules have been calculated by Jarmain (I7-12407), and a new determination of the molecular constant for some bands of the $\mathrm{CH}$ molecule has been made by Kiess (I7-4409r). A rotational analysis of the $9 \cdot 6 \mu$ telluric ozone band has been performed by Migeotte.

The wave-lengths of the violet band of the $\mathrm{CN}$ molecule have been measured by Weinard ( 17 -44093), and an improved set of molecular constants has thus been obtained.

Measurements of the wave-lengths of one band due to the $\mathrm{CH}$ molecule have been performed by Kiess (I7-12433), and an improved identification of the molecule in the solar spectrum has been made.

\section{TABLES OF ASTROPHYSICAL INTEREST}

The new edition of the Rowland tables prepared by Mrs Moore-Sitterly in collaboration with Utrecht Observatory is now ready for the spectral region 8700-3500 $\AA$. It is hoped that the whole project will be terminated before the Moscow General Assembly of the I.A.U. An important contribution has been received from the Dunsink Observatory where Mrs Brück and Dr Thompson photographed the ultra-violet spectrum up to $3020 \AA$ and obtained a complete intensity record of their plates.

A table of the solar spectrum between II 984 and $25578 \AA$ has been published by Mohler (I7-5IIII). A photometric atlas of the solar spectrum extending from 2.8 to $23.7 \mu$, resulting from tracings of the solar spectrum taken at the Jungfraujoch Observatory, has been published by Migeotte and collaborators ( $17-51$ IOg). The atlas is composed 


\title{
COMMISSION 12
}

of two parts: the first contains the reproductions of the solar tracings, whereas the second contains the wave-length measurements and identifications of elements and compounds.

Tables of dissociation constants for ten molecules formed by the elements $\mathrm{H}, \mathrm{C}, \mathrm{N}$ and $O$ have been computed by Pecker (I8-680I6) for many values of temperature. Tables of the partition function of $\mathrm{H}$ and Fe II have also been computed by the same author.

At Arcetri, Noci has computed similar tables of the partition function of the element $\mathrm{Na}$.

Tables of the Planck radiation function have been computed by $\mathrm{McD}$ onald ( $16-63547$ ) for a large number of temperatures extending from $15000^{\circ}$ to $50000^{\circ} \mathrm{K}$.

\section{ACKNOWLEDGEMENTS}

The writer wants to thank all members of the Commission for their collaboration in sending the information which has been a great help for the preparation of this report. To the members of the staff of the Arcetri Observatory, the writer is very grateful for the valuable help and assistance given during the selection and assimilation of the material contained in this report. Particular thanks are due to Mr Forbes who has helped in reading and revising the manuscript.

\author{
G. R I GH I I \\ President of the Commission
}

President: G. Righini.

Report of Meeting. I4 August I958

Secretary: Miss E. A. Müller.

Before asking for approval of the Draft Report the President read the comments on the report he had received in personal letters from several members of the Commission.

After a short discussion the Draft Report was then approved by all members of the Commission.

Dr Moore-Sitterly and Prof. Minnaert reported on the progress of the new Rowland Tables of the Solar Spectrum.

Showing a page of the tables, already in press, as a sample Dr Moore-Sitterly pointed out that the wave-lengths of many faint lines had been re-measured and improved. Upto-date values of the excitation potentials are included in the tables.

The identification of atomic lines is fairly complete, whereas the identification of molecular lines is being studied and revised by Dr Moore-Sitterly and Dr Broida, and will be completed at a later date. It is planned to publish separate tables for each individual molecular band. Prof. Minnaert reported on the determination of the equivalent widths of all the lines in the solar spectrum, a work carried out by the Utrecht astronomers during the last Io years and now almost completed, the last part in the ultra-violet to be finished shortly. He discussed briefly the choice of the continuous background in the ultra-violet region, letting it increase gradually from the visible region up to the Balmer limit. At the end of his account Prof. Minnaert remarked that anyone who is interested in the equivalent width of any Fraunhofer line in the solar spectrum may write to the Utrecht Observatory for information; he ended his report by thanking the I.A.U. for the subsidies granted for the publication of the new Solar Spectroscopic Tables.

The President thanked Prof. Minnaert and Dr Moore-Sitterly for the enormous amount of work that they have carried out in this most valuable new edition of the Rowland Tables.

The next paper on the agenda was an investigation into the double reversal of the $\mathrm{H}$ and $\mathrm{K}$ lines of $\mathrm{Ca}$ II in the solar spectrum by $\mathrm{L}$. Goldberg, O. C. Mohler, and E. A. Müller, presented by Dr Goldberg. The profiles of the $\mathrm{H}$ and $\mathrm{K}$ line cores were obtained photoelectrically with the varuum spectrograph of the McMath-Hulbert Observatory at dif- 


\section{RADIATION ET SPECTROSCOPIE SOLAIRE}

ferent points on the solar disk from $\mu=\mathrm{I} \cdot 0$ to $\mu=0 \cdot 2$. At the centre of the disk the central intensities of $\mathrm{H}$ and $\mathrm{K}$ are $2.95 \%$ and $2.36 \%$ respectively, in terms of the neighbouring continuous spectrum near $\lambda 4000$. At $\mu=0.2$ the central intensities are $2.4 \mathrm{I} \%$ and $2.0 \mathrm{I} \%$ for $H$ and $K$, respectively, also with reference to the continuum at $\lambda 4000$ at the centre of the disk. The relative intensity ratio $\mathrm{H}: \mathrm{K}$ is found to be constant in the inner absorption core and nearly the same for all values of $\mu$. This suggests a source function that varies linearly with the logarithm of $N_{h}$, the number of Ca II atoms per $\mathrm{cm}^{2}$ above height, $h$, in the chromosphere. The Doppler widths were derived by two methods, namely (I) by an inter-compurison method which is independent of the source function, and (2) by using the depth dependence of the source function and the shapes of the profiles. The Doppler widths thus found increase from $0.063 \AA$ at $\mu=\mathrm{I} \cdot 0$ to $0 \cdot$ Iro $\AA$ at $\mu=0 \cdot 2$. The derived Doppler widths and source function are employed to calculate the profiles of the inner cores of $\mathrm{H}$ and $\mathrm{K}$. Extremely good agreement is found between the calculated and the observed central cores at all five values of $\mu$. The variation of the Doppler widths from centre to limb is discussed, and it is concluded that the widening of the profiles to the limb is caused by an increase of turbulence with height combined with anisotropy. The comparison of the Doppler widths derived for $\mathrm{Ca}$ II with results previously obtained for the $\lambda$ Io 830 line of He I supports the hypothesis that the chromosphere is heterogeneous and is composed of regions of quite different physical properties. (The detailed paper will be published in $A p . J$.

Following Dr Goldberg's account Dr V. L. Khokhlova reported on her investigation of the $\mathrm{H}_{3}$ and $\mathrm{K}_{3}$ lines in the solar spectrum. Her study is based on photographic plates of about $0.2 \AA / \mathrm{mm}$ dispersion obtained with the Crimean solar tower spectrograph. By determining the total half-width of the $\mathrm{K}_{3}$ and $\mathrm{H}_{3}$ adsorption an upper limit for the turbulent velocity $v_{t} \leqslant 8 \mathrm{~km} / \mathrm{sec}$ was derived. The total half-width of the $\mathrm{H}_{3}$ and $\mathrm{K}_{3}$ absorption is 0.19 $\AA$ at the solar centre and $0.34 \AA$ at $\sin \theta=0.99$, and this widening towards the limb is explained by assuming that the number of absorbing ions along the line of sight varies proportional to $\sec \theta$. Good agreement between calculated and observed values was found for $v_{t}=6.7 \mathrm{~km} / \mathrm{sec}$. The height of formation of $\mathrm{K}_{3}$ and $\mathrm{H}_{3}$, defined as the height where $\tau\left(\mathrm{K}_{3}\right)=\mathrm{I}$, was calculated for $v_{t}=20$, I5 and $8 \mathrm{~km} / \mathrm{sec}$, using the Ca II concentration derived from eclipse observations. It was found that the height at which $\mathrm{K}_{\mathbf{s}}$ and $\mathrm{H}_{3}$ are formed is at about $4500-5000 \mathrm{~km}$ and that it is practically independent of the value of $v_{t}$. The analysis of the turbulent velocities obtained by different authors shows that the turbulent velocities for the helium lines and those for the metallic lines differ systematically by a factor of 2 for the same heights. This confirms the conclusion by V. A. Krat and other authors that in the layer where helium is formed the temperature or the turbulent velocity or both are higher than in the layer where the metals are formed.

A short discussion followed, mainly in connexion with the paper presented by Dr Goldberg. Dr Houtgast suggested that the turbulence may be associated with the spicules and that the increase of turbulence with height in the chromosphere could possibly be explained by the gas motions in the spicules. To a question by Dr Athay on the meaning of turbulence in association with spicules, whether it refers to the motions of the spicules themselves or to motions in a smaller scale within the spicules, Dr Houtgast replied that he thinks the motions of the gases in the lower part of the spicules are laminar and that at the top of the spicules a turbulent motion develops. In this connexion Dr Athay reported on some results obtained from studies of the profiles of the $\mathrm{H} \alpha, \mathrm{H} \beta$, and $\mathrm{D}_{3}$ lines of spicules observed with the Climax coronograph. A comparison of $\mathrm{H} \alpha$ profiles with $\mathrm{D}_{3}$ profiles gives a kinetic temperature of $65000^{\circ}$ and zero turbulent velocity. A comparison of $\mathrm{H} \beta$ with $\mathrm{D}_{3}$ gives a kinetic temperature of $55000^{\circ}$ and a turbulent velocity of $6.5 \mathrm{~km} /$ sec. In both cases, the turbulent velocity seems to be very small in comparison with the thermal velocity. Dr Waddell, addressing Dr Goldberg, mentioned that the dependence of the turbulence upon depth and angle is complicated by the fact that much of the turbulent energy could be concentrated between the scale of the micro-turbulence, the basis on which the observations were analysed, and the large-scale turbulence revealed by the different types of profiles obtained at slit lengths of one minute of arc. A further 


\section{COMMISSION 12}

comment was made by Dr Jeffreys in recalling that a similar type of source-function, which was used in a study of prominences, turned out to be incorrect, the resulting temperatures being considerably in error.

Dr Goldberg, in answering the different comments and questions, pointed out that the profiles of the $\mathrm{H}$ and $\mathrm{K}$ line cores used are averaged profiles and that it is planned to study profiles over smaller regions observed with a smaller slit photographically rather than photo-electrically. The variation of the turbulence with height is small as compared to the variation with angle. It is not known whether the $H$ and $K$ line cores originate in the spicules or outside, but it seems probable that $\mathrm{Ca}$ II originates in the cool and $\mathrm{He}$ in the hot regions. The $\mathrm{H}$ and $\mathrm{K}$ lines are formed in heights above $2000 \mathrm{~km}$, but to fix the height definitely one must know the model of the chromosphere. So far this question has not been solved, and the published models vary by over a factor of Ioo.

Dr Thomas then reported on an investigation carried out by himself and Dr Jeffreys concerning source-functions in the chromosphere considering the non-coherent to coherent scattering processes. With a source-function of particular algebraic expression and six parameters to be chosen it can be shown that for a reasonable looking set of parameters one can calculate line profiles with emission reversals. Dr Jeffreys suggests that at a certain distance from the line centre one should take a frequency-dependent source-function to reproduce line profiles of the kind shown in the paper presented by Dr Goldberg.

Dr Ohman explained briefly a new technique in operation at the Swedish station in Anacapri for measuring magnetic field strengths and polarities. The method of observing the polarized components in a Zeeman pattern with a Savart plate $(A p . J$. IIr, 362, I950) gave good results when a spectrograph having large dispersion $(0.5 \mathrm{~mm} / \AA$ or more) was employed. The method was found less satisfactory for smaller dispersions and in order to increase the sensitivity, so that also smaller dispersion could be used, the following improved arrangement has been developed at the Swedish Astrophysical Station in Anacapri.

By placing a Savart plate (angular separation of fringes about $15 \mathrm{~min}$ of arc) behind an achromatized Ramsden eyepiece (focal length $40 \mathrm{~mm}$ ), and by using as analyser a Rochon prism (giving two beams with a separation of about $15 \mathrm{~min}$ of arc), two superimposed but slightly displaced spectra are observed. If the polariscope is adjusted in such a way that the interference fringes are perpendicular to the spectral lines, and if the light is allowed to traverse a quarterwave plate before entering the polariscope, the circularly polarized Zeeman components produce wave-shaped spectral lines. By measuring the maximum distances $D_{1}$ and minimum distances $D_{2}$ between corresponding waves in the double image, the Zeeman displacement $\Delta \lambda$ is obtained as

$$
\Delta \lambda=\mathbf{I} / 4\left(D_{1}-D_{2}\right) \text {. }
$$

This shows that the method is twice as sensitive as the original arrangement with single spectral lines. The fact that two superimposed spectra are being observed does not seem to introduce a noticeable drawback. By placing a slit in the focal plane in front of the eyepiece, transmitting only a narrow strip of the spectrum and selecting in this way the proper line only, a higher contrast may be obtained. But in this case the setting is more difficult, and experience has shown that the method using superimposed spectra is in general more convenient. In order to determine the polarities of the fields a narrow strip of polaroid is placed in front of the eyepiece.

Next Dr Stepanov reported on weak magnetic fields ( $H<$ Iooo oersted) measured on records of $5 \times 5 \mathrm{sec}$ of arc resolving power obtained with the Babcock type magnetograph of the Crimean Astrophysical Observatory. Over undisturbed regions the general character of the magnetic field remains unchanged for one to two hours; however, one can observe an appreciable change in position even after $15 \mathrm{~min}$ in regions of weak fields ( $\mathrm{I}-3$ oersted). The filaments of fine chromospheric structure observed in $\mathrm{H} \alpha$ tend to run parallel to the lines of force. The 'streams' of these filaments connect the sunspot fields and local fields with four regions of opposite polarity. Such connexions can be traced between regions separated by up to $300000 \mathrm{~km}$ and between magnetic regions in opposite hemi- 


\section{RADIATION ET SPECTROSCOPIE SOLAIRE}

spheres. W. E. Stepanov and N. N. Petrova found that the $\mathbf{K}_{\mathbf{2 3 2}}$ emission in flocculi increases with field strength up to a certain value (corresponding to the fields $20<H<200$ oersted) and then decreases steadily with field strength. The maximum value of a magnetic field corresponds to the central dark part of a ring-shaped bright flocculus observed on $\mathrm{K}$-spectroheliograms. This suggests that, perhaps, the formation of flocculi is connected with the absorption of hydromagnetic waves in the chromosphere with energy densities of about $10^{2}-\mathrm{IO}^{4} \mathrm{ergs} / \mathrm{cm}^{3}$.

Prof. Severny mentioned briefly that photo-electric records of magnetic fields in some sunspots were obtained recently at the Crimean Astrophysical Observatory with the solar magnetograph of high resolving power (about $I \times I$ sec of arc). The records show clearly the 'pips' corresponding to very strong concentrations of lines of force in areas of several seconds of arc in diameter.

Then Dr Bumba presented some preliminary results of his investigations of magnetic fields in unipolar sunspots and of the Evershed effect. From the magnetic maps and from the Zeeman splitting it is found that all sunspots have both magnetic polarities. The lineof-sight component of the field intensity in the region of the weaker polarity increases from only a few per cent of the main field intensity near the solar centre to about $40 \%$ in a distance of $50^{\circ}$ from the solar centre. The interpretation is that, assuming the magnetic field of a sunspot has the form proposed by Cowling, one looks through its lines of forces in various directions. Assuming a certain height of formation of the $\mathrm{Ca}$ II lines above a spot and using the Zeeman splitting in $\mathrm{K}_{2}$ and $\mathrm{H}_{2}$ maps of the vertical distribution of the magnetic field above a spot may be constructed. Typical asymmetries of the lines in the region of the umbra and especially of the penumbra are observed and called 'flags'. The behaviour of the 'flags' particularly in the vicinity of the $H$ and $K$ line centres may be explained by a large velocity gradient in a thin layer of the solar atmosphere. The study of the geometry of movements and of the magnetic fields leads to the preliminary conclusion that the movements in the sunspots occur along the lines of force of the magnetic fields.

Answering Prof. Kiepenheuer's question about conclusions drawn from observations which were mainly longitudinal, Prof. Severny points out that in $80 \%$ of the observed cases it was found that the filaments run perpendicular to the iso-gauss. To Dr Goldberg's question whether the magnetic maps were made in the $\mathrm{K}$ line Prof. Severny answered that the magnetic maps were made in the line $\lambda 5_{250} \AA$, and that, due to the similarity of these maps and the $\mathrm{K}$-spectroheliograms, it was concluded that the magnetic fields reach into the chromosphere.

The last account was given by Dr Giovanelli who spoke briefly about a new technique developed in Canberra to detect magnetic fields in the chromosphere. The device is not yet fully developed and the calibration is difficult and not yet solved, but no spectrograph is required, since this magnetograph uses a Lyot filter with a Wollaston prism replacing the polaroid.

Thanking all the speakers, the President adjourned the meeting. 